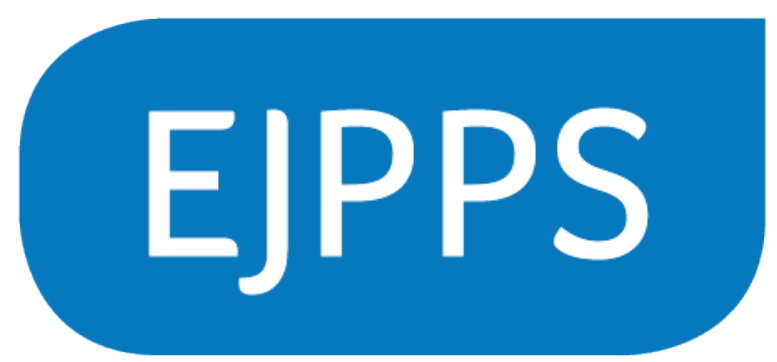

\begin{tabular}{|l} 
EUROPEAN JOURNAL OF \\
PARENTERAL AND \\
PHARMACEUTICAL SCIENCES
\end{tabular}

EJPPS - European Journal of Parenteral and Pharmaceutical Sciences Volume 25 Issue 3

https://www.ejpps.online/annex21importationofmedicinalprod

https://doi.org/10.37521/ejpps25302

Opinion Paper

Annex 21: Importation of Medicinal Products

Colin Newbould Wasdell Group

Corresponding Author: Colin Newbould, Wasdell Group

Email: colinnewbould@phss.co.uk 


\section{Annex 21: Importation of Medicinal Products}

This short review is aimed at looking at how Annex 21 fits within the current GMP guidance's outlined in Eudralex "The Rules Governing Medicinal Products in the European Union Volume 4 EU Guidelines for Good Manufacturing Practice for Medicinal Products for Human and Veterinary Use".

As described in the annex the legal basis for publishing the detailed guidelines is derived from "Article 47 of Directive 2001/83/EC on the Community code relating to medicinal products for human use and Article 51 of Directive 2001/82/EC on the Community code relating to veterinary medicinal products. And this document provides guidance for the interpretation of the principles and guidelines of good manufacturing practice (GMP) for medicinal products as laid down in Directive 2003/94/EC for medicinal products for human use and Directive 91/412/EEC for veterinary use".

In short, it covers both human and veterinary products and yet, unlike Annex 16, which provides significant detail more pronounced to the duties and responsibilities of the Qualified Person, this newly proposed Annex offers a simple overview and outlines clear expectations and practices which should be adopted within a Quality System for Importation activities of medicinal products.

The new annex clearly references other relevant chapters and annexes within the Eudralex guidance and helps draw together the principles and basis of these good practices to a focal point.

But why have another Annex when the guidance already exists? The key reasons probably sit with the emphasis placed on the responsibilities of the marketing authorisation holder (MAH) throughout the relationship. Mentioned only once in Annex 16 and yet four times in this new annex, this Annex makes it clear that the MAH holds the responsibility for placing medicinal products on the market.

There are certainly some helpful clarifications and expectations placed on Product Quality Reviews and which party should be undertaking these which again will help ensure the final certifying QP has appropriate oversight of the whole process and supply chain. Additionally, there are expectations that analytical results are compared between the importation testing sites and those of the thirdparty manufacturing facilities. These reviews should include investigation of any trends and discrepancies. There is an expectation that the certification site is ensuring there is an ongoing stability program and more importantly the full information of the program including the protocols, results, reports should be available at the facility responsible for certification.

One of the more controversial changes which also impacts perhaps on arrangements in Quality Agreements and legacy supply chains will be the requirement to have access to full batch documentation at all times. It is expected that the frequency of the QP review of this information is justified and defined within the Quality Management System. Again, this is standard practice, but it certainly helps reinforce the requirement that the MAH must play a key role in ensuring the QPs involved in certification are furnished with the ability to undertake their roles adequately.

One aspect of the Annex which could cause some controversy is around section 5.3 which describes Batch documentation, including batch certificates, supplied by the third country manufacturing sites. The section talks about the language should be understood by the importer. This then suggests that it may be necessary to provide documents in more than one language to facilitate understanding.

Although this sounds simple enough, in practice might prove somewhat more difficult to achieve. It would seem by way of example to ask operators (or machines) to create dual or even multiple language versions of batch documents at time of manufacture. It is difficult enough to get operators to "get it right first time" in their own language after all.

So perhaps more guidance as to how this could be achieved may be needed, for example 
- Multiple language versions (text) but only single data input boxes for the numerical values (even this is awkward if you consider non Arabic numeral systems or even the different use of commas (,) and full stops / decimal points (.) within even the EU Member states). By way of examples, one thousand may be written as 1,000 or 1000 and occasionally this is written in some states as 1.000 . so, a required input of say $1250 \mathrm{~g}$ might possibly be recorded by the operator or automated system as $1,250 \mathrm{~g}, 1.250 \mathrm{~g} 1250 \mathrm{~g}$ or $1.25 \mathrm{~kg}$ More rarely 1,0 has been written to mean 1.0. The certifying QP will need to interpret these statements as to what quantity has actually been input

- Use of a blank certified Master batch doc in the language of the importer / certifying QP with version number linking to the original batch document etc

This simple Annex cover all aspects of importation including GDP, sampling, and stability. Although it could conceivably seem repetitive to those conversant in GMP and working with already excellent supply chain arrangements, it now clearly reinforces the responsibility of the MAH to ensure they are aware of the requirements needed by final certifying QP and MIA holder. It holds a number of good clarifications. Areas for further clarification will be needed around the language of communication with the documentation but in the spirit of the guidance it does seem logical that anyone reviewing documents should be able to understand them. 